\title{
Incidental subclavian artery injury during right internal jugular vein catheterization via ultrasonography
}

\author{
Suyoeng Cho ${ }^{1}$, Jeong-Il Choi ${ }^{2}$, Seongtae Jeong ${ }^{1}$ \\ 'Department of Anesthesiology and Pain Medicine, Chonnam National Unviersity Medical School and Hwasun Hospital, \\ ${ }^{2}$ Department of Anesthesiology and Pain Medicine, Chonnam National Unviersity Medical School and Hospital, Gwangju, Korea
}

Received June 16, 2020

Revised June 29, 2020

Accepted June 30, 2020

Corresponding author

Seongtae Jeong

Department of Anesthesiology and

Pain Medicine, Chonnam National

Unviersity Medical School and

Hwasun Hospital, 42 Jebong-ro,

Dong-gu, Gwangju 61469, Korea

Tel: +82-62-220-6895

Fax: +82-62-232-6294

E-mail: anesjst@jnu.ac.kr

ORCID:

https://orcid.org/0000-0002-6245-9779
Subclavian artery injury during central venous catheterization appears to be reported less than in actual cases. We report management of incidental subclavian artery catheterization in a 65-year-old man who has limited neck motion, undergoing emergency spine hematoma removal surgery. Despite the use of ultrasonography for central venous catheterization via right internal jugular vein, incidental subclavian artery injury has not been prevented. The management of incidental arterial injury during central venous catheterization is discussed, and a review of the literature is provided. To prevent injury to the right subclavian artery during right IJV catheterization, the location of adjacent blood vessels should be ascertained and not damaged. It is important to note that the damage can also occur due to ultrasonography. When right subclavian artery injury is suspected, the catheter should not be removed. Early detection of injury and early intervention may reduce morbidity and mortality.

Keywords: Central venous catheterization; Subclavian artery injury; Ultrasonography; Interventional radiotherapy

\section{INTRODUCTION}

Various complications such as arterial puncture, hemothorax, and pneumothorax can occur during central venous catheterization [1]. Unintended artery puncture is the most common complication reported in $3 \%$ to $15 \%$ of patients undergoing central venous catheterization. Carotid artery is the most common arterial puncture site. Complications related to large vessel damage are reported to be less than $1 \%$. However, subclavian artery injury appears to be reported less than in actual cases [2,3]. Recently, the clinical application of ultrasonography has increased with insertion of central venous catheter. In order to prevent various complications, especially vascular injury, ultrasound is recommended in all such cases if possible [4]. However, despite the use of ultrasonography, the risk of arterial puncture remains. We present an incidental subclavian artery injury during right internal jugular vein (IJV) catheterization using ultrasonography, which was successfully treated with interventional radiation therapy.

\section{CASE REPORT}

A 65-year-old man $(70 \mathrm{~kg}, 175 \mathrm{~cm})$ with epidural hematoma extending from L2 to S1 was admitted to emergency room because of decreased lower extremity motor grade. He was scheduled to undergo emergent laminotomy and hematoma removal. Upon arrival at the operating room, the 
patient's neck was restricted in both flexion and extension movements in physical examination. Routine monitoring of the patient included electrocardiography (EKG), pulse oximetry, and blood pressure measurements. After anesthesia induction, an arterial cannulation of the left radial artery was performed for continuous blood pressure monitoring. The patient was then positioned for right IJV catheterization.

We placed a pillow on the patient's back and adopted a Trendelenburg position, with the head held approximately $10 \mathrm{~cm}$ above the bed because of his rigid neck. Therefore, additional cotton pads were placed under his head. At the cricoid cartilage level at the point where the sternal head and clavicular head of sternocleidomastoid muscle meet, we

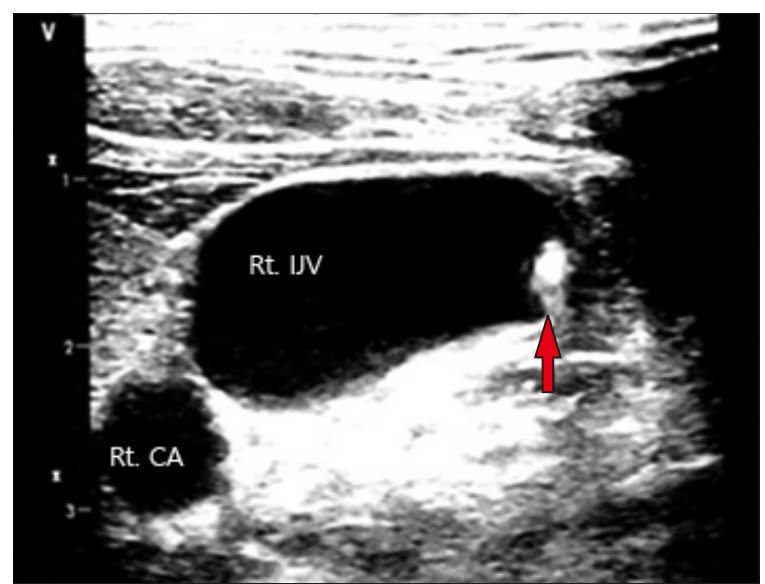

Fig. 1. Ultrasonography finding. Central venous catheter is in the right internal jugular vein under out-of-plane view. Arrow shows the catheter. CA: carotid artery, IJV: internal jugular vein.
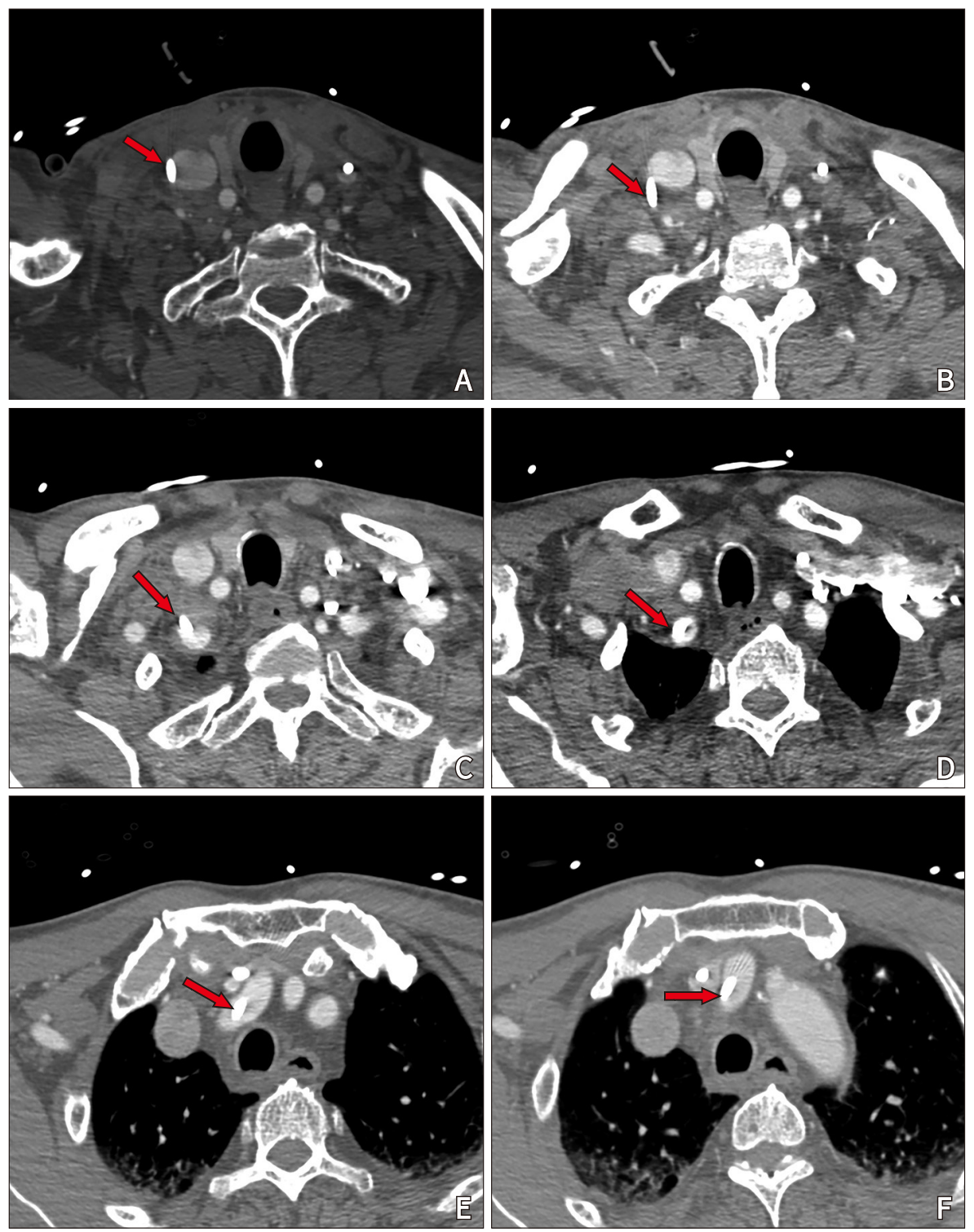

Fig. 2. CT-scan findings. (A) Central venous catheter in the right internal jugular vein (IJV). (B) Catheter is out of right IJV. (C, D) Catheter is in the right subclavian artery. ( $E$, F) Catheter is in the brachiocephalic trunk. Arrows show the catheter. 
used ultrasonography to confirm the location of carotid artery and right IJV. The retrograde flow of dark and nonpulsatile blood via the puncture needle was confirmed under ultrasonography guidance via the anterior approach using the Seldinger's technique. Using ultrasound in out-of-plane approach, we adjusted the position of the puncture needle while assuring the puncture needle position in the right IJV. Dark and nonpulsatile blood was aspirated, so the guidewire was threaded through a puncture needle without resistance. We ensured that the guidewire was in the right IJV using out-of-plane ultrasonography. We routinely evaluated the guidewire in the IJV via in-plane ultrasonography before the insertion of tissue dilator. However, it was not possible to determine the guidewire location via in-plane approach due to limited space for probe rotation because the patient's neck was too short and firm in flexion. After tissue dilation for catheter insertion, Edwards oximetry central venous catheter (8.5F/3L, Edward Lifesciences Technology Sarl, Puerto Rico, US) was inserted over the guidewire. After finishing the catheter insertion, we connected the central venous pressure (CVP) monitoring line to the catheter. CVP monitoring showed not venous wave but arterial wave form. At this point, we re-evaluated catheter position using ultrasonography. The out-of-plane approach using ultrasonography, at the cricoid cartilage level, revealed the catheter location in the right IJV (Fig. 1). When the ultrasonography probe was moved to the right clavicle level, the catheter was not appeared to be in the right IJV or subclavian vein. Catheter was suspected to be in the artery which is parallel to vein (this vessel was proved right subclavian artery by CTscan; Fig. 2).

We evaluated around the right-side neck. No visible hematoma or swelling was observed. In addition, there was not any hemodynamic or respiratory parameter changes. After conversation with surgeon, we decided that it is better to proceed the scheduled spine surgery than further evaluation or treatment of the catheter.

We inserted an additional central catheter in the left IJV via ultrasonography whereas keeping the right IJV catheter. The operation was completed without any problems. No pneumothorax or hemothorax was observed in the chest AP obtained immediately after surgery (Fig. 3). The patient was transferred to the surgical intensive care unit.

Neck CT angiography on postoperative day (POD) 1

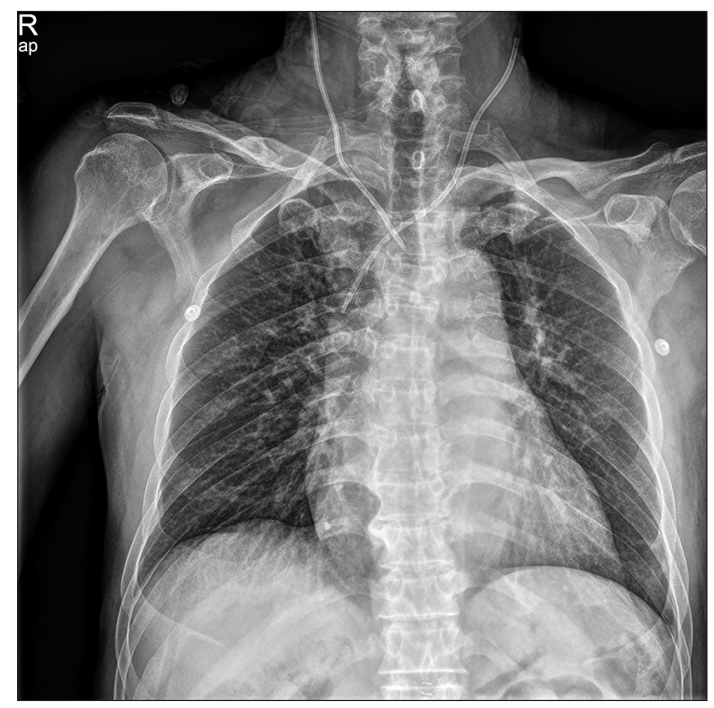

Fig. 3. Chest A-P view. There was no pneumothorax or hemothorax. Right internal jugular catheter tip is suspected in the brachiocephalic trunk. Left internal jugular catheter tip is in the superior vena cava.

showed that the catheter passed through the right IJV and right subclavian artery to the origin of brachiocephalic trunk (Fig. 2). We removed the right IJV catheter with interventional radiotherapy on POD3. In the right brachiocephalic trunk angiogram, it was confirmed that the catheter was inserted in the right subclavian artery without extravasation of the contrast media. The balloon was placed at the point of catheter insertion. The compression was performed for 3 min using a balloon catheter (Fig. 4). In a follow-up angiogram, a pseudoaneurysm was detected in the right subclavian artery, which required compression for 5 minutes using a balloon catheter. After about $10 \mathrm{~min}$, the intervention was completed after no further extravasation of contrast media was observed (Fig. 4). The patient had no further complications in a follow-up evaluation.

\section{DISCUSSION}

Several cases of right subclavian artery injury during right IJV catheterization have been reported, although it is unclear how the central catheter injures the right subclavian artery. In some cases, it is damaged by the guide wire [5,6]. In other cases, it may be damaged by the dilator device [2] or puncture needle $[7,8]$. In our case, we speculated that the puncture needle probably injured the right subclavian artery. Initially, the puncture needle was inserted into the right IJV, but the needle apparently went farther and penetrated 

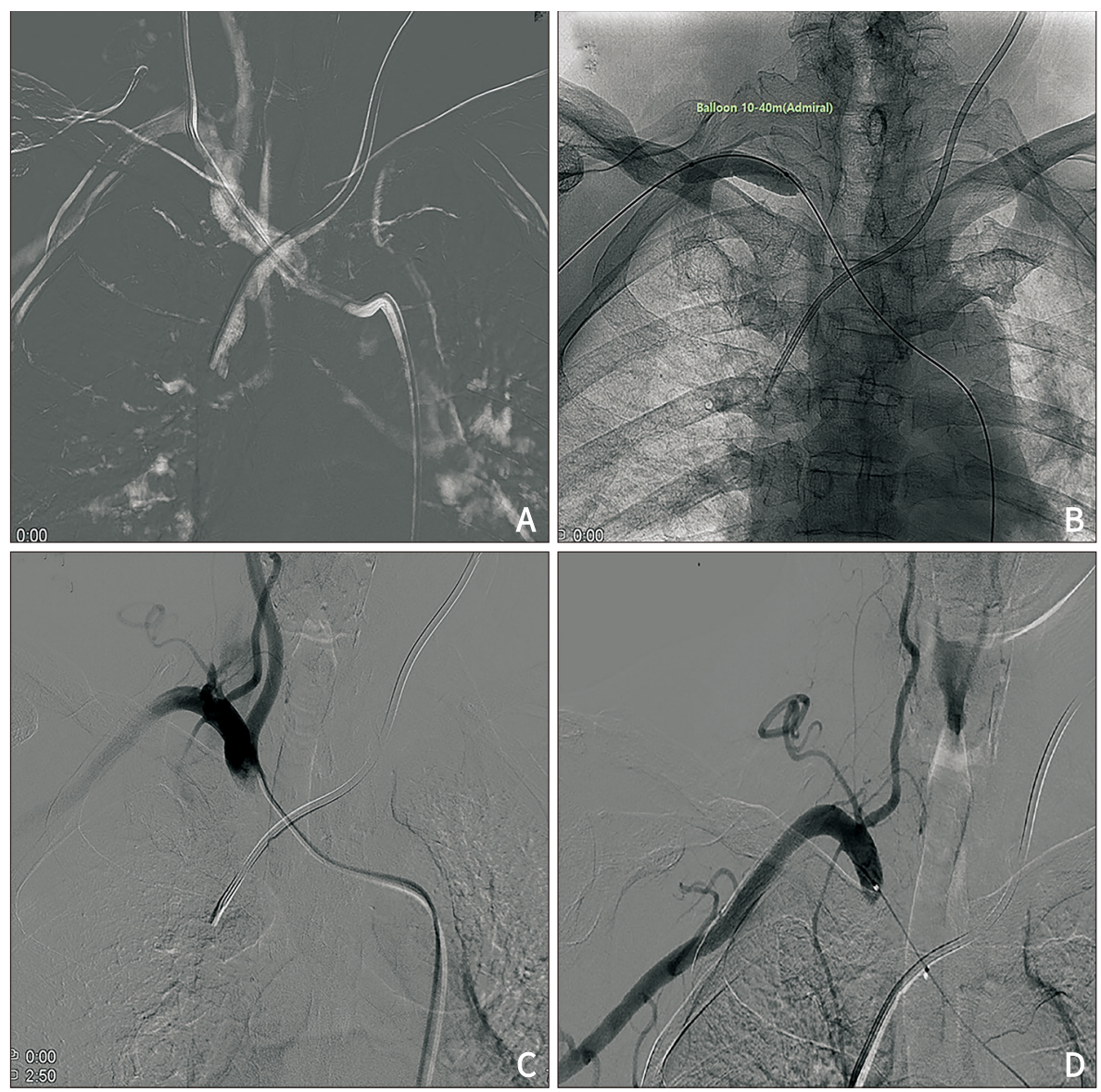

Fig. 4. Radiologic findings during interventional radiotherapy. (A) Central venous catheter is in the right subclavian artery. There is no extravasation of contrast media. Chest (B) $\mathrm{X}$-ray after catheter removal. Balloon catheter (Admiral) is in the subclavian artery. (C) There is a pseudoaneurysm at subclavian artery in follow-up angiogram. (D) There is no extravasation after additional compression.

the posterior wall of the right IJV through the guide wire. If the needle was inserted too deep, it punctured the IJV wall and injured the adjacent right subclavian artery. In our case, presumably the right subclavian artery was punctured because the patient's neck was too short and the needle was inserted far lower than the anatomical landmark. Furthermore, the needle was inserted laterally into the right IJV, and not from the center (Fig. 1). Therefore, the puncture needle or dilator may have penetrated through the right IJV into the right subclavian artery located medially.

Kulvatunyou et al. [2] provided an anatomical explanation for this complication because the right subclavian artery branches arise from the brachiocephalic trunk medial and posterior to the internal jugular vein. Whereas the left subclavian artery originates from the aortic arch and passes laterally to the left IJV, the proximal position of the right subclavian artery and IJV overlap (Fig. 5). Kulvatunyou et al. designated the event as the 'right sided phenomenon'. Acute hypotension during right IJV catheterization with evidence of hemothorax suggests possible injury to the right subclavian artery. Several precautions are needed to prevent this complication. First, because of the anatomical relationship between the right IJV and the right subclavian artery, the puncture needle should be cephalad to the apex of the triangle formed by the two heads of the sternocleidomastoid muscle. Under ultrasound guidance, especially in the short-necked patient, even if the position of the vessels was confirmed using the ultrasound probe, the actual site of needle entry should be higher than the probe position. We should also recognize the surface anatomical landmark, and puncture the right IJV at the center of the lumen. Second, before applying the dilator device, the position of guide wire should be determined. To ensure that the guide wire is located close to the brachiocephalic vein from the right IJV, any inadvertent catheterization of adjacent artery can be prevented by tracing the ultrasound probe down to the brachiocephalic vein. Third, in order to prevent the risk of serious arterial injury, the dilator device should be advanced only until the subcutaneous tissue [9].

When arterial puncture due to less than 20-gauge needle 


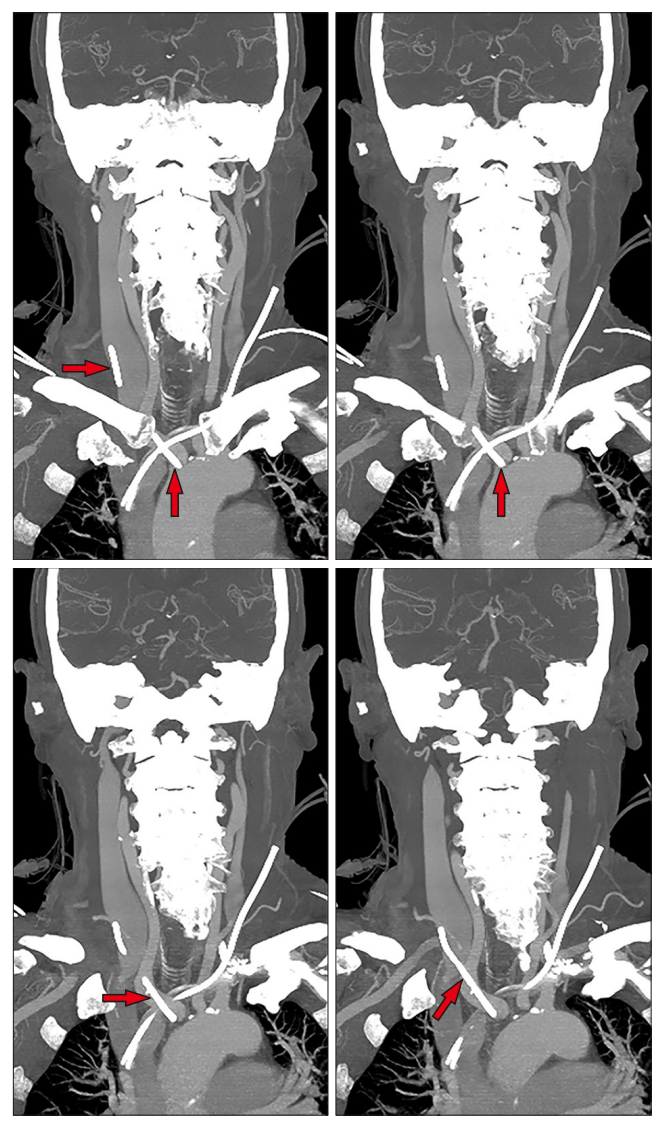

Fig. 5. Neck CT findings. The catheter inserted into the right internal jugular vein entered the brachiocephalic trunk through the overlapping right subclavian artery.

is suspected during central venous catheterization, extraction and external compression for $10 \mathrm{~min}$ can be safely carried out. However, if there is a large-bore catheter injury ( $>7$ French), early recognition of arterial trauma and prompt management are required. The pull/pressure technique is associated with a significantly higher morbidity than surgical or endovascular management, including stroke, hematoma causing airway compression, false aneurysm, or death. If the site of suspected arterial injury is easily accessible surgically, it is desirable to leave the catheter in place and perform open exploration and direct arterial repair. If the injured site is hard to surgically access, it is desirable to ensure that the catheter is in place and consider percutaneous treatment with a covered stent or percutaneous closure device [10].

In our case, there were no hemodynamic changes or hemothorax. Considering the neurological complications following epidural hematoma, we decided to proceed with the planned surgery, and the catheter was safely removed via postsurgical interventions because we detected arterial injury early and did not remove the catheter. If the catheter had been removed, the patient would have suffered severe hypotension and hemothorax.

Ultrasonography guidance is used to safely puncture venous and arterial structures. The use of two-dimensional ultrasound reduces the rate of total complications, arterial punctures, and the chances of hematoma formation, compared with techniques based on anatomical landmarks. It increased the success rate and the chances of success during the first attempt. It also decreased the number of attempts for successful cannulation and time to successful cannulation [11]. However, the bony structures of anterior chest wall and the infraclavian area are not visible via ultrasonography, and it is difficult to detect subclavian artery injury and also avoid complications [5].

In conclusion, to prevent injury to the right subclavian artery during right IJV catheterization, the location of adjacent blood vessels should be ascertained and not damaged. It is important to note that the damage can also occur due to ultrasonography. When right subclavian artery injury is suspected, the catheter should not be removed. Early detection of injury and early intervention may reduce morbidity and mortality.

\section{CONFLICT OF INTEREST}

No potential conflict of interest relevant to this article was reported.

\section{REFERENCES}

1. Gropper MA, Cohen NH, Eriksson LI, Fleisher LA, Leslie K, Wiener-Kronish JP. Miller's anesthesia. 9th ed. Philadelphia: Elsevier, Inc.; 2020. p. 1170.

2. Kulvatunyou N, Heard SO, Bankey PE. A subclavian artery injury, secondary to internal jugular vein cannulation, is a predictable right-sided phenomenon. Anesth Analg 2002;95:564-6, table of contents.

3. Vemuri NN, Narendra PL. Critical upper limb ischemia due to thrombus in the right subclavian artery: An uncommon complication of right internal jugular vein cannulation. Anesth Essays Res 2015;9:101-4.

4. Apfelbaum JL, Rupp SM, Tung A, Connis RT; American Society of Anesthesiologists Task Force on Central Venous Access. Practice guidelines for central venous access: reply. Anesthesiology 2020;133:242-3. 
5. Tokue H, Tsushima Y, Morita H, Endo K. Successful interventional management for subclavian artery injury secondary to internal jugular catheterization: a report of two cases. Cardiovasc Intervent Radiol 2009;32:1268-71.

6. Beilin Y, Bronheim D, Mandelbaum C. Hemothorax and subclavian artery laceration during " $\mathrm{J}$ " wire change of a right internal jugular vein catheter. Anesthesiology 1998;88:1399400.

7. Lee DJ, Yun JC, Choi HR, Im UJ, Woo SH. Subclavian artery perforation and hemothorax after right internal jugular vein catheterization. Korean J Anesthesiol 2013;64:558-9.

8. Que DD, Liu L, Song XD, Wang XB, Zhang XL, Zhou YJ, et al. Flexible subclavian artery closure for an inadvertent injury to the internal mammary artery during internal jugular vein catheterization. Chin Med J (Engl) 2016;129:868-70.

9. Bowdle A, Jelacic S, Togashi K, Ferreira R. Ultrasound identification of the guidewire in the brachiocephalic vein for the prevention of inadvertent arterial catheterization during internal jugular central venous catheter placement. Anesth Analg 2016;123:896-900.

10. Guilbert MC, Elkouri S, Bracco D, Corriveau MM, Beaudoin N, Dubois MJ, et al. Arterial trauma during central venous catheter insertion: Case series, review and proposed algorithm. J Vasc Surg 2008;48:918-25; discussion 925.

11. Brass P, Hellmich M, Kolodziej L, Schick G, Smith AF. Ultrasound guidance versus anatomical landmarks for internal jugular vein catheterization. Cochrane Database Syst Rev 2015;1:CD006962. 